\title{
Latest developments in the field of stem cell research and regenerative medicine compiled from publicly available information and press releases from nonacademic institutions in December 2018
}

\author{
Ilic Dusko*,1 \\ ${ }^{1}$ Stem Cell Laboratories, Guy's Assisted Conception Unit, Department of Women \& Children's Health, Faculty of Life Sciences \& \\ Medicine, King's College London, SE1 9RT, London, UK \\ *Author for correspondence: dusko.ilic@kcl.ac.uk
}

First draft submitted: 24 January 2019; Accepted for publication: 12 February 2019; Published online: 19 March 2019

\section{Business development \\ Collaborations, partnerships \& alliances \\ Co-development agreement: Gilead \& Agenus}

Gilead (CA, USA; www.gilead.com) and Agenus (MA, USA; www.agenusbio.com) announced today the companies have entered into an immuno-oncology partnership focused on the development and commercialization of up to five novel immuno-oncology therapies. Under the terms of the agreement, Agenus will receive US $\$ 150$ million upon closing, which includes a US $\$ 120$ million upfront cash payment and a US $\$ 30$ million equity investment. The agreement also includes approximately US $\$ 1.7$ billion in potential future fees and milestones. Gilead will receive worldwide exclusive rights to AGEN1423, which has an estimated Investigational New Drug (IND) filing by year-end 2018. Gilead will also receive the exclusive option to license two additional programs: AGEN1223 and AGEN2373. Agenus has filed the IND for AGEN1223 and has an estimated IND filing for AGEN2373 in the first half of 2019. Agenus will be responsible for developing the option programs up to the option decision points, at which time Gilead may acquire exclusive rights to the programs on option exercise.

\section{License agreement: ERS Genomics \& Lonza}

ERS Genomics (Ireland; www.ersgenomics.com) and Lonza (Switzerland; www.lonza.com) have announced today a license agreement to provide Lonza with access to ERS Genomics' CRISPR/Cas9 genome editing technology patents. Lonza will have the ability to utilize CRISPR/Cas9 in its bioproduction products and services and also for use in induced pluripotent stem cells (iPSC) for research applications.

\section{License agreement: Fate \& Max Delbrünck Center}

Fate Therapeutics (CA, USA; www.fatetherapeutics.com) has secured an exclusive option to an intellectual property portfolio owned by the Max Delbrück Center for Molecular Medicine (Germany; www.mdc-berlin.de) covering novel humanized chimeric antigen receptor (CAR) constructs that uniquely and specifically bind B-cell maturation antigen (BCMA). Under the agreement with Max Delbrünck Center, Fate Therapeutics holds an exclusive option to exclusively license the portfolio for all cell products, including CAR NK- and T-cell products, derived from iPSCs.

\section{License agreement: Pluristem \& Chart}

Pluristem Therapeutics (Israel; www.pluristem.com) has entered into a license agreement with a subsidiary of Chart Industries (GA, USA; www.chartindustries.com) regarding Pluristem's thawing device for cell-based therapies. Per the terms of the agreement, Chart obtains the exclusive rights to manufacture and market the thawing device in all territories worldwide, excluding China, with Pluristem receiving royalties from sales of the product and supply 
of an agreed number of devices. Pluristem's point-of-care thawing device technology is designed to allow for the precise and automated thawing of cells in a controlled and monitored environment and is expected to result in the highest levels of cell viability and quality. The technology includes many advanced unique proprietary features which were designed to result in the leading thawing devices on the market.

Strategical agreement: Interxon \& Merck

Intrexon Corporation (MD, USA; www.dna.com) and its wholly owned subsidiary Precigen, have announced agreement with Merck (Germany; www.merck.com) for the development of CAR-T-cell therapies. Under the terms of the agreement, Merck will assign its exclusive CAR-T development rights to Intrexon, allowing Precigen to continue autonomous development of its proprietary CAR-T technology platform. Merck will receive US $\$ 150$ million in Intrexon stock in exchange for assigning its CAR-T rights.

\section{Launching new products, services...}

\section{Allele}

After 3 years of cleanroom construction and 2 years of quality system establishment, Allele Biotech (CA, USA; www.allelebiotech.com) has produced using mRNA reprogramming technology its initial batch of six clinical-grade iPSC lines from fibroblast stocks that are fully current Good Manufacturing Practice (cGMP)-compliant.

\section{Prellis}

Tissue engineering company Prellis Biologics (CA, USA; www.prellisbio.com) launched the first ever line of prevascularized 3D tissue scaffolds. The scaffolds are biocompatible and can be used by researchers to grow any cell type in 3D formats $1000 \times$ larger than 3D spheroids. Among Prellis' Vascular Tissue Blanks are the prevascularized 3D Tissue Chip ${ }^{T M}$, Organoids ${ }^{T M}$ and the Vascular Bundle ${ }^{T M}$. The beta test product Do-It-Yourself Organ on a $\mathrm{Chip}^{\mathrm{TM}}$ is designed for experienced microfluidics labs to set up their own cell-cell interfaces for toxicology screening with flow across a $20 \mu \mathrm{m}$ interface. Alveolus ${ }^{\top \mathrm{M}}$, another beta product, acts as a human lung surrogate structure; it is a single alveolus surrounded by a bed of human-sized capillaries and is being offered to those interested in the air-liquid interface of the lung. Prellis has developed the first holographic printing system able to match and accurately replicate human organ and tissue structures for R\&D and organ transplantation. The combined resolution and speed of Prellis Biologics' printing technology allows for full human organ systems to be created with cell-compatible biomatrices.

\section{Clinical trials \\ Bellicum}

Bellicum Pharmaceuticals (TX, USA; www.bellicum.com) has reported safety and promising activity of BPX-601 in Part 1 of a Phase I/II dose-escalation study in patients with advanced, metastatic pancreatic cancer expressing prostate stem cell antigen (PSCA). A total of 12 patients with advanced metastatic pancreatic cancer expressing PSCA were treated with escalating doses of BPX-601 cells in a $3+3$ design. Nine of 12 patients received a single dose of rimiducid following BPX-601 treatment to evaluate its effect on cell expansion and persistence. Patients in the study received a reduced conditioning regimen comprising cyclophosphamide only. Four out of six efficacy-evaluable patients treated with BPX-601 and a single dose of rimiducid had stable disease, with two patients demonstrating tumor shrinkage greater than $20 \%$. No cytokine release syndrome or neurotoxicity of any grade was reported. BPX-601 is a novel GoCAR- ${ }^{\circledR}$ cell candidate incorporating Bellicum's inducible MyD88/CD40 (iMC) co-activation domain, designed to boost $\mathrm{T}$-cell proliferation and persistence via administration of rimiducid. BPX-601 is being evaluated as a treatment for solid tumors expressing PSCA, including pancreatic, prostate and gastric cancer.

\section{Celgene}

Celgene (NJ, USA; www.celgene.com) has announced initial safety data from its ongoing proof-of-concept trial of JCARH125 in patients with relapsed/refractory multiple myeloma (https:/clinicaltrials.gov; ID: NCT03430011). JCARH125 is an investigational BCMA-targeting CAR T-cell therapy being developed by Juno Therapeutics (WA, USA; www.junotherapeutics.com), a Celgene Company. The data reported from the multicenter, Phase I/II EVOLVE trial includes patients who have been treated with JCARH125 in the dose escalation study. The primary objectives of the Phase I portion of the trial are safety and identification of a recommended Phase II dose. The 
patients enrolled in the study had to have received at least three prior lines of multiple myeloma therapy, including an autologous stem cell transplant for transplant eligible patients, a proteasome inhibitor, an immunomodulatory drug and an anti-CD38 monoclonal antibody. Dose escalation is currently ongoing. In this first report of JCARH125 data, the median follow-up was only 11 weeks, yet among infused patients, the overall response rate was $82 \%$. At the lowest dose level of $50 \times 10^{6}$ CAR T cells, the overall response rate was 79 and $43 \%$ of patients achieved stringent complete response or complete response.

\section{Celgene \& Bluebird}

Celgene (NJ, USA; www.celgene.com) and Bluebird (MA, USA; www.bluebirdbio.com) have announced initial data from the ongoing Phase I clinical study of bb21217 (CRB- 402), an investigational next-generation anti-BCMA CAR T-cell therapy being studied in patients with relapsed/refractory multiple myeloma (https://clinicaltrials.gov; ID: NCT03274219). bb21217 is an investigational anti-BCMA CAR T-cell therapy that uses the bb2121 CAR molecule with a manufacturing process designed to improve CAR T-cell functional persistence. bb2121 and bb21217 are bluebird bio's lead investigational anti-BCMA CAR T therapies being developed in collaboration with Celgene. Patients included in these preliminary Phase I results $(n=12)$ had a median age of 63 years (min; max: 44-69 years). They had received a median of seven prior lines of therapy (min; max: 4-17 lines) and 83\% of patients received a prior autologous stem cell transplant, whereas $58 \%(n=7)$ of patients had high-risk cytogenetics. All treated patients received a dose of $150 \times 10^{6} \mathrm{CAR}^{+} \mathrm{T}$ cells. The median follow-up after bb21217 infusion was 26 weeks (min; max: 4-51 weeks). Eight of the 12 patients (67\%) treated with bb21217 developed cytokine release syndrome; four Grade 1, three Grade 2, one Grade 3 case and no Grade 4 cases. Additionally, three of the 12 patients (25\%) experienced neurotoxicity, including one Grade 1, one Grade 2 and one Grade 4 case. All CRS and neurotoxicity events resolved and no deaths occurred on study. Of the 12 patients who received treatment with bb21217, 83\% ( $\mathrm{n}=10)$ achieved an objective clinical response by the International Myeloma Working Group Uniform Response Criteria for Multiple Myeloma. As of the data extract, responses are ongoing in nine out of ten patients, including three with a complete response or stringent complete response, two with a very good partial response and four with partial response.

\section{Cynata}

Cynata Therapeutics (Australia; www.cynata.com) has completed the Phase I clinical trial of CYP-001 for the treatment of steroid-resistant acute graft-versus-host disease (GvHD). CYP-001 is Cynata's lead Cymerus ${ }^{\top \mathrm{M}}$ iPSCderived mesenchymal stem cell (MSC) product candidate (https://clinicaltrials.gov; ID: NCT02923375). The Phase I trial of CYP-001 represents the first time a clinical trial using iPSC-derived therapy has been completed.

Participants were adults who had undergone an allogeneic hematopoietic stem cell transplant (HSCT) to treat a hematological (blood) disorder and subsequently been diagnosed with steroid-resistant grade II-IV GvHD. The first eight participants were enrolled in Cohort A and received two infusions of CYP-001 at a dose of 1 million cells $/ \mathrm{kg}$, up to a maximum dose of 100 million cells. Seven participants in Cohort B received two infusions of CYP-001 at a dose of 2 million cells $/ \mathrm{kg}$, up to a maximum dose of 200 million cells. There was 1 week between the two CYP-001 infusions in each participant.

The trial's primary objective was to assess the safety and tolerability of CYP-001, while the secondary objective was to evaluate the efficacy of two infusions of CYP-001 in adults with steroid-resistant GvHD. The primary evaluation period concluded 100 days after the first dose in each participant. Efficacy was assessed on the basis of response to treatment (as determined by change in GvHD grade) and overall survival at 28 and 100 days after the administration of the first dose. After the completion of the primary evaluation period, participants entered a longer-term, noninterventional follow-up period, which will continue for up to 2 years after the initial dose.

Key highlights of the final results from the primary evaluation period are:

- Overall response rate by Day 100 was 87\%; 13 out of 15 patients showed an improvement in GvHD severity by at least one grade compared with baseline;

- Complete response rate by Day 100 was 53\% GvHD signs and symptoms completely resolved in 8 out of 15 patients;

- Overall survival at Day 100 was at least $87 \%$;

- No treatment-related serious adverse events or safety concerns were identified. 


\section{Gamida}

Gamida Cell (Israel; www.gamida-cell.com) has reported translational data showing that recipients who received $\mathrm{NiCord}^{\circledR}$, an investigational cell therapy in Phase III clinical development for allogeneic hematopoietic bone marrow stem cell transplant had rapid and robust reconstitution of key immune cells (https://clinicaltrials.gov; ID: NCT02730299). Key findings from the analysis include the following:

- $91 \%$ of patients achieved successful immune reconstitution of $\mathrm{CD}^{+} \mathrm{T}$ cells at 100 days after transplantation with NiCord.

- Immune reconstitution of $\mathrm{T}$ cells was similar in the NiCord group (median age: 41.5 years) compared with the younger cohorts receiving unmanipulated cord blood and unrelated bone marrow (median ages 15.4 and 14.3 years, respectively).

- Immune reconstitution of B cells $(\mathrm{p}=0.02)$ and NK cells $(\mathrm{p}<0.001)$ was significantly faster after transplantation with $\mathrm{NiCord}$ compared with the other groups.

- Immune reconstitution after NiCord transplantation was associated with recovery of a broad spectrum of T-cell, B-cell and NK-cell subsets representing a range of effector functions similar to that observed with other graft sources.

Results from the multicenter Phase I/II clinical study evaluating the safety and efficacy of NiCord as a standalone transplant showed that the patients transplanted with NiCord had rapid and durable engraftment of neutrophils and platelets, as well as prompt immune reconstitution [1]. The median time to neutrophil recovery was shortened by nearly $50 \%$ for patients who received NiCord compared with a retrospective cohort of patients who received standard umbilical cord blood. NiCord also demonstrated an acceptable safety profile for patients undergoing bone marrow transplant.

\section{iCell}

iCell Gene Therapeutics (NY, USA; www.icellgene.com) has announced results from a study ongoing at Chengdu Military General Hospital of ICG144, the first CLL1-CD33 Compound CAR T-cell (cCAR-T) in clinical study, in patients with particularly difficult to treat acute myeloid leukemia (AML). Patients 1 and 2 both failed multiple previous cycles of therapy and presented with complex conditions limiting further options. Treatment with CLL1CD33 cCAR led both patients to complete response and engraftment of haploidentical stem cell transplantation (allo-HSCT) without myeloablative conditioning. CLL1-CD33 cCAR is a compound Chimeric Antigen Receptor immunotherapy with two distinct functional CAR molecules expressed on a $\mathrm{T}$ cell, directed against the surface proteins CLL1 and CD33. The diseases treated by CLL1-CD33 cCAR could include acute myeloid leukemia, myelodysplastic syndromes, chronic myeloid leukemia and chronic myeloproliferative neoplasms. CLL1 is associated with leukemia stem cells and disease relapse, while CD33 is expressed on bulky AML disease. Treatment of AML is a challenge due to heterogeneity of AML bearing cells, which renders single antigen targeting CAR T-cell therapy ineffective. ICG1 44 cCAR is designed to target the mechanisms of single-CAR relapse, specifically antigen escape and leukemic stem cells.

\section{Regulations, approvals, acquisitions... Green light BrainStorm}

BrainStorm Cell Therapeutics (NY, USA; www.brainstorm-cell.com) has announced that the US FDA (MD, USA; www.fda.gov) has accepted the IND application to initiate a Phase II clinical trial of NurOwn ${ }^{\circledR}$ for the treatment of progressive multiple sclerosis. NurOwn are produced from autologous, bone marrow-derived MSCs that have been expanded and differentiated ex vivo. MSCs are converted into MSC-NTF cells by growing them under patented conditions that induce the cells to secrete high levels of neurotrophic factors.

\section{Mustang Bio}

Mustang Bio (NY, USA; www.mustangbio.com) has announced that the FDA (MD, USA; www.fda.gov) granted Orphan Drug Designation to MB-102 for the treatment of blastic plasmacytoid dendritic cell neoplasm (BPDCN), a rare and incurable blood cancer with a median survival of less than 18 months and no standard of care. MB-102 is a CAR T-cell therapy that is produced by engineering patient $T$ cells to recognize and eliminate CD123-expressing 
tumors. CD123 is widely expressed on bone marrow cells of patients with myelodysplastic syndromes, as well as in hematologic malignancies including AML, B-cell acute lymphoblastic leukemia, hairy cell leukemia, BPDCN, chronic myeloid leukemia and Hodgkin's lymphoma. In the first-in-human clinical trial, MB-102 has demonstrated complete responses at low doses in AML and BPDCN without dose-limiting toxicity (https://clinicaltrials.gov; ID: NCT02159495).

\section{Novartis}

Novartis (Switzerland; www.novartis.com) has announced that the therapeutic goods administration (TGA; Australia; www.tga.gov.au) has approved Kymriah ${ }^{\circledR}$ (tisagenlecleucel, formerly CTL019) CAR T-cell therapy. The approved indications are for the treatment of pediatric and young adult patients up to 25 years of age with B-cell precursor acute lymphoblastic leukemia that is refractory, in relapse post-transplant, or in second or later relapse; and for the treatment of adult patients with relapsed or refractory diffuse large B-cell lymphoma after two or more lines of systemic therapy.

\section{XenoTherapeutics}

The US FDA (MD, USA; www.fda.gov) has cleared XenoTherapeutics (MA, USA; www.xenotherapeutics.org) to begin a first-of-its-kind human clinical trial that uses topically applied live skin cells derived from genetically engineered pigs as a treatment for severe burns (https://clinicaltrials.gov; ID: NCT03695939). The biologically active Xeno-Skin product comprises dermal and epidermal tissue layers, sterilized and sourced from animals designed to be free of the viral pathogens that have been an obstacle to the use of porcine xenotransplants in the past, the company said. The transplanted skin aims to provide temporary coverage as an immediate treatment option for patients with extensive deep-partial and full-thickness burn wounds. XenoTherapeutics is planning to enroll six patients at Massachusetts General Hospital (MA, USA; www.massgeneral.org) in a single-arm trial.

Mergers, acquisitions...

Novartis \& CellforCure

Following an earlier agreement with CellforCure (France; www.cellforcure.com) to produce CAR-T-cell therapies including Kymriah (tisagenlecleucel), Novartis (Switzerland; www.novartis.com) has announced an offer to acquire the company. CellforCure, a French company, is one of the first and largest contract development and manufacturing organizations producing cell and gene therapies in Europe. After acquisition, CellforCure would become wholly owned Novartis manufacturing facility.

Novartis \& Endocyte

Novartis (Switzerland; www.novartis.com) has completed the acquisition of Endocyte (IN, USA; www.endocyte.c om), a biopharmaceutical company focused on developing radioligand and CAR-T therapies for cancer treatment, known for its Lu-PSMA-617, a potential first-in-class radioligand therapy in Phase III development for metastatic castration-resistant prostate cancer (https://clinicaltrials.gov; ID: NCT03511664).

\section{Capital market \& finances \\ Cynata}

Cynata Therapeutics (Australia; www.cynata.com) has received from the Australian National Health and Medical Research Council (NHMRC; https://nhmrc.gov.au) a grant to fund a Phase II clinical trial to evaluate Cynata's Cymerus $^{\top M}$ iPSC-derived MSCs as a treatment for osteoarthritis. The aim of the Phase II clinical trial is to assess the effect of Cymerus MSCs compared with placebo on clinical outcomes and knee joint structure over a 2-year period, in 448 patients with osteoarthritis of the knee. Preclinical research has shown that MSCs can exert a number of important effects that may improve outcomes in patients with osteoarthritis, including release of cytokines and growth factors that reduce inflammation and promote tissue repair, new blood vessel formation and regeneration of compromised cartilage.

\section{Cellect \& Cell2in}

The Korea-Israel Industrial R\&D Foundation (Korea; www.koisra.co.kr) has approved grant for the collaboration between Cellect Biotechnology (Israel; www.cellect.co) and Cell2in (Korea; www.cell2in.com), providing financing for the joint project. Cellect will use Cell2in proprietary FreSHtracer technology for the functional identification of 
stem cells. Both companies will collaborate on a proof-of-concept of combining technologies in order to significantly improve stem cells selection process. Cellect is aiming to show more efficient quantification, as compared with current industry standards of MSCs and hematopoietic stem cells (HSCs), and validation through quantification that Fas Ligand (FasL), Cellect's main active ingredient, accelerates MSC expansion relative to processes currently used in approved products as well as products in clinical trials.

\section{Emulate}

Emulate Bio (MA, USA; https://emulatebio.com) has received a US\$ 2 million research grant from the NIH's National Center for Advancing Translational Sciences (MD, USA; https://ncats.nih.gov) to use the company's Intestine-Chip to conduct experiments that will provide insights into the function of the intestinal barrier and its response to bacterial invasion in microgravity. Experiments will be conducted on the International Space Station (FL, USA; www.issnationallab.org) and on Earth to analyze cellular interactions within the Intestine-Chip and to better understand the impact of bacterial challenges on gastrointestinal homeostasis. The Intestine-Chip is designed to emulate the complexity of a human intestine in a living microengineered environment, which includes the intestinal epithelium, endothelial cells, resident immune cells, bacteria and enteric sensory neurons. The other partner is Space Tango (KY, USA; https://spacetango.com), a company that designs, builds and operates facilities onboard the International Space Station for research and manufacturing.

\section{Financial \& competing interests disclosure}

The author has received an honorarium from Future Science Group for the contribution of this work. The author has no other relevant affiliations or financial involvement with any organization or entity with a financial interest in or financial conflict with the subject matter or materials discussed in the manuscript apart from those disclosed.

No writing assistance was utilized in the production of this manuscript.

\section{References}

1. Horwitz ME, Wease S, Blackwell B et al. Phase I/II study of stem-cell transplantation using a single cord blood unit expanded ex vivo with nicotinamide. J. Clin. Oncol. 37(5), 367-374 (2018). 